\title{
Increasing the Performance of the Iterative Computed Tomography Image Reconstruction Algorithms
}

\author{
Shimaa Abdulsalam Khazal ${ }^{1}$, Mohammed Hussein Ali $^{2^{*}}$
}

\section{Authors affiliations: \\ 1) Electronic and \\ Communications Engineering, \\ Al-Nahrain University, \\ Baghdad-Iraq. \\ shimaa9191@gmail.com}

$\left.2^{*}\right)$ Electronic and

Communications Engineering,

Al-Nahrain University,

Baghdad-Iraq.

malhavani@gmail.com

\section{Paper History:}

Received: $13^{\text {th }}$ Oct. 2019

Revised: 29th Dec. 2019

Accepted: $16^{\text {th }}$ July 2020

\begin{abstract}
Computed tomography (CT) imaging is an important diagnostic tool. CT imaging facilitates the internal rendering of a scanned object by measuring the attenuation of beams of $\mathrm{X}$-ray radiation. CT employs a mathematical technique of image reconstruction; those techniques are classified as; analytical and iterative. The iterative reconstruction (IR) methods have been proven to be superior over the analytical methods, but due to their prolonged reconstruction time, those methods are excluded from routine use in clinical applications. In this paper the reconstruction time of an IR algorithm is minimized through the employment of an adaptive region growing segmentation method that focuses the image reconstruction process on a specified region, thus ignoring unwanted pixels that increase the computation time. This method is tested on the iterative algebraic reconstruction technique (ART) algorithm. Some phantom images are used in this paper to demonstrate the effects of the segmentation process. The simulation results are executed using MATLAB (version R2018b) programming language, and a computer system with the following specifications: CPU core i7 $(2.40 \mathrm{GHz})$ for processing. Simulation results indicate that this method will reduce the reconstruction time of the iterative algorithms, and will enhance the quality of the reconstructed image.
\end{abstract}

Keywords: Computed Tomography, ART, Shepp-Logan, Iterative Reconstruction, Seeded Region Growing.

$$
\begin{aligned}
& \text { زيادة أداء خوارزميات إعادة بناء الصور المقطعية التكرارية } \\
& \text { شياء عبد السلام خزعل ، محمد حسين علي } \\
& \text { التصوير المقطعي (CT) هو أداة تشخيصية ضرورية. التصوير المقطعي يسهل عملية التصوير الداخلي للجسم }
\end{aligned}
$$

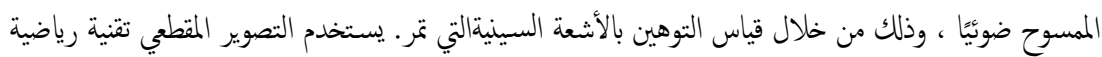

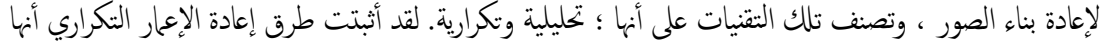

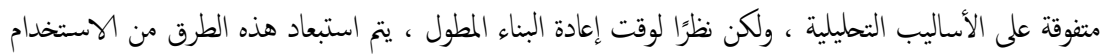

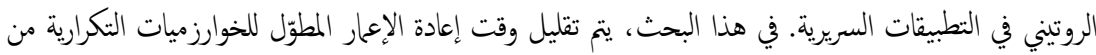

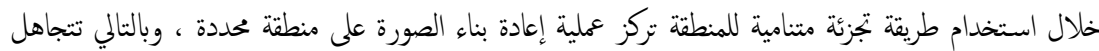

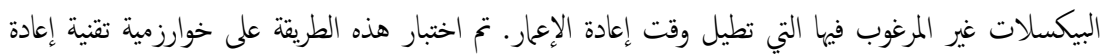

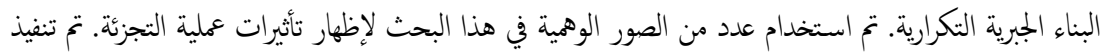

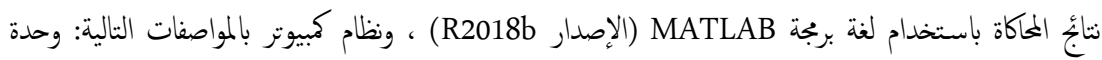

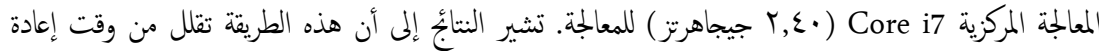

$$
\begin{aligned}
& \text { البناء للخوارزميات التكرارية ، وتعزز جودة الصورة المعاد بناؤها. }
\end{aligned}
$$

\section{Introduction}

Tomography signifies an imaging process that constructs cross-sectional images, which reveal the internal structure of a scanned object in a nondestructive manner [1]. While the diagnostic advances of CT are well- recognized, the associated hazards of increased exposure to ionizing radiation has become an area of increasing concern [2]. The

NJES is an open access Journal with ISSN 2521-9154 and eISSN 2521-9162

This work is licensed under a Creative Commons Attribution-NonCommercial 4.0 International License 
use of analytical reconstruction techniques or what is commonly known as the FBP algorithm in CT image reconstruction has been orthodox for many years. However, it poses many limitations, such as the production of possible streak artifacts and a prominent increase in image noise levels if the radiation dose is reduced exceedingly [3]. The recent appearance of IR techniques in CT poses as a replacement that allows a reduction in radiation dose while sustaining the quality of the reconstructed image [4]. The first CT scanners in the early 1970s used iterative reconstruction algorithms. Yet, they were not used clinically, due to insufficiency in computational power. The iterative reconstruction algorithms became commercially available for the first time in 2009. Since then, this technique has modernized the field of radiology [5]. Although this approach is theoretically simpler than the analytical approaches, it requires more computational complexity. However, iterative methods improve the quality of the image when it is not feasible or when the number of acquired projections is diminutive. In addition, this approach accomplishes better image quality when the projections of X-ray beams are not uniformly distributed over the scan trajectory [6]. In 2007, Tessa Van Hemelryck et al. [7] utilized the iterative ART algorithm with two other iterative techniques. Their work allotted a lot of time for speeding up these algorithms, beginning with the creation of the weight matrix. The algorithms implementations and calculations were carried out in MATLAB. The authors faced some challenges with the creation of the weight matrix and the implementation of the algorithms. They found out that these algorithms require a long time to process, which led to the use of small phantom images to compensate for the limitations of the workstation. In 2009, Yufei Chen et al. [8] suggested an efficient method for the automatic segmentation of CT images. The results of the segmentation reviled the efficiency and effectiveness of their proposed method. In 2010, Martin A. Brooks [9] selected five of the fundamental algebraic reconstruction algorithms for extensive testing. The quantitative numerical results obtained in this study showed that algebraic techniques could actually produce an image from corrupt and/or missing data. The author concluded that further refinement of algebraic techniques might ultimately lead to a breakthrough in CT. In 2011, Nihad Mesanovic et al. [10] proposed a segmentation technique to accurately segment sections of CT images, which can help the radiologist in the early diagnosing of diseases, the technique of region growing employed in their research proved to be effective in doing so. In 2012, Shilpa Kamdi and R.K.Krishna [11] prepared a survey discussing some image segmentation methods. Their research considered the problems and issues in the area of image segmentation research, and the use of those techniques in different studies. Their research showed that the region growing segmentation method offers several advantages over the other conventional segmentation techniques. In 2015, Chandni Panchasara and Amol Joglekar [12] presented some segmentation algorithms, discussing their advantages and properties. In 2015, Noor Hussein Fallooh [13] prepared a detailed study on the image reconstruction algorithms for both types of methods; iterative and analytical. The obtained result was compared with a different number of iterations and different measurements of image quality. In 2017, MarieLouise Aurumskjöld [14] evaluated and optimized IR methods in CT regarding image quality and radiation dose. The author included a combination of studies on phantoms and patients and showed that IR algorithms have different strengths and weaknesses, but the important conclusion from all studies in this work is that, all IR algorithms improve image quality, noise, signal-to-noise ratio, and contrast-to-noise ratio compared to the FBP algorithm for the same radiation dose. In 2018, Brahim AIT SKOURT, Abdelhamid EL HASSANI, and Aicha MAJDA [15] proposed a lung CT image segmentation. The advantage of the method presented in this work is the fact that it could be applied to a wide area of different medical image segmentation tasks. In 2019, Hiroki Kawashima et al. [16] evaluated the physical image quality using IR algorithms and compared the outcomes with those using the FBP algorithm. Their research showed that IR algorithms achieved notable noise reduction and preserved the resolution.

In this paper; the timing of the iterative ART algorithm reconstruction process is reduced by constraining its reconstruction of a phantom CT image to be performed on a user-defined region, this region is generated using the seeded region growing segmentation process, and the obtained results are compared with the traditional algorithm before applying this acceleration approach.

\section{The Iterative CT Image Reconstruction}

Iterative reconstruction techniques have a general structure; they model the forward projections of the original CT device acquisition, by assuming an initial guess image. Those forward projections are compared with the measured projections acquired from the CT device to calculate the difference, which leads to an update. Thus, improving the image until the difference between the forward projections and the measurements is minimized. Iterative algorithms work by simply solving a system of linear equations [17], which is achieved by first discretized the image into pixels as described in Fig. 1 [1] [18].

In this figure $(x)$ represents the image pixels, and the projections are $(p)$, they could be related via linear equations as shown by Eq. (1) [1]:

$$
\begin{aligned}
& x_{1}+x_{2}+x_{3}=p_{1} \\
& x_{4}+x_{5}+x_{6}=p_{2} \\
& x_{7}+x_{8}+x_{9}=p_{3} \\
& x_{3}+x_{6}+x_{9}=p_{4} \\
& x_{2}+x_{5}+x_{8}=p_{5} \\
& x_{1}+x_{4}+x_{7}=p_{6} \\
& 2(\sqrt{2}-1) x_{4}+(2-\sqrt{2}) x_{7}+2(\sqrt{2}-1) x_{8}=p_{7} \\
& (\sqrt{2}) x_{1}+(\sqrt{2}) x_{5}+(\sqrt{2}) x_{9}=p_{8} \\
& 2(\sqrt{2}-1) x_{2}+(2-\sqrt{2}) x_{3}+2(\sqrt{2}-1) x_{6}=p_{9}
\end{aligned}
$$




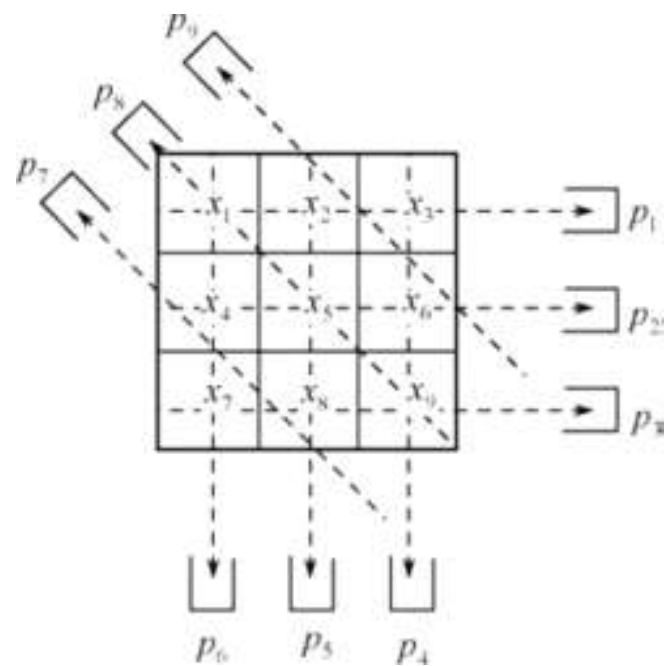

Figure (1): A linear equations system [1].

This system could be re-written in the matrix form as demonstrated by Eq. (2) [1]:

$$
A X=P \ldots(2)
$$

Where $X=\left[x_{1}, x_{2}, \ldots, x_{9}\right]^{T}, P=$ $\left[p_{1}, p_{2}, \ldots, p_{9}\right]^{T}$, and $(A)$ is the coefficient matrix of the system. If the inverse matrix $\left(A^{-1}\right)$ of $(A)$ exists, the reconstructed image is given by Eq. (3) [1].

$$
X=A^{-1} P \ldots(3)
$$

The significant size of the matrix $(A)$ prevents its storage in a computer system, so this matrix is created in a row-by-row manner only. Any iterative method that modifies $(A)$ could not be utilized. Instead, methods that employ matrix $(A)$ and its transposed matrix $\left(A^{-1}\right)$ are used [1]. Mathematically this means that all iterative reconstruction (IR) algorithms evaluate the reconstruction process starting with the first estimate of the image $(x)$, which is assumed to be zeros, this value is updated in a manner that relies on the IR algorithm employed.

The conjecture of IR algorithms is founded on six important stages that are shown in Fig. 2. Those steps follow the CT projection measurements; the first step is to generate an initial guess image, then the simulated projections are generated. The next step compares the simulated projection data with the projections measured from the CT scanner. If any discrepancies occur, the estimated image is corrected based on the update equation of the utilized IR algorithm. This process of correcting the image is replicated, until the satisfaction of a condition that was predefined by the algorithm. The final image is generated when that condition is gratified [19].

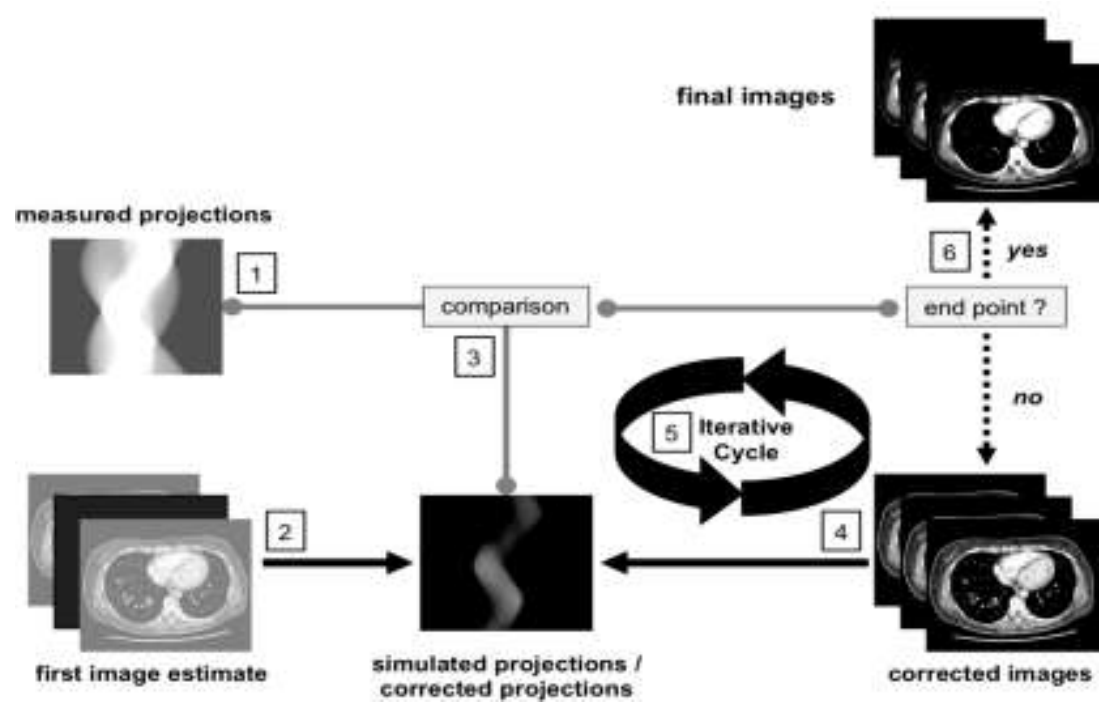

Figure (2): The basic steps of IR algorithms [5].

\subsection{ART Algorithm}

The principle of the ART algorithm (also known as the Kaczmarz method) is to make the estimated image satisfy one equation at a time [1]. Historically, the ART algorithm was the first algorithm applied in CT. This algorithm is executed one projection ray at a time, and the image is updated after each ray is considered. Symbolically, the algorithm could be written as demonstrated in Eq. (4) [1]. $x^{\text {next }}=x^{\text {current }}-$

Backproject $_{\text {ray }}\left\{\frac{\text { Project }_{\text {ray }}\left(x^{\text {current }}\right)-\text { Measurement }_{\text {ray }}}{\text { Normalization Factor }}\right\}$
In this equation $\left(x^{\text {current }}\right)$ refers to the initial guess image, $\left(x^{\text {next }}\right)$ refers to the updated initial guess image, $\left(\right.$ Project $\left._{\text {ray }}\left(x^{\text {current }}\right)\right)$ are the simulated projections of the initial guess image, Measurement $_{\text {ray }}$ ) are the measured projections data acquired from the CT scanner, and (Normalization Factor) is a matrix consisting of (1's).

ART algorithm function by using the information of only one beam sum (i.e. projection ray) at a time as shown in Fig. 3. It modifies the initial image by summing the pixels along some straight path and comparing this sum to the measured projection along that ray. The difference between the calculated projections obtained from the image estimate and the 
projections measured from the passing $\mathrm{X}$-ray beam is used to update the initial image. Therefore, ART is occasionally titled the ray-by-ray reconstruction method [20].

Figure 3 shows a projection beam $\left(P_{i}\right)$ of width $(\Delta \xi)$, passing through the tissue .The pixel size is given by $(b)$. The weight $\left(a_{i j}\right)$ is thus determined by Eq. (5) [20].

$$
\begin{array}{r}
\text { aij }=(\text { illuminated area of pixel } j \text { by ray } I) \\
/(\text { total area of pixel } j) \ldots(5)
\end{array}
$$

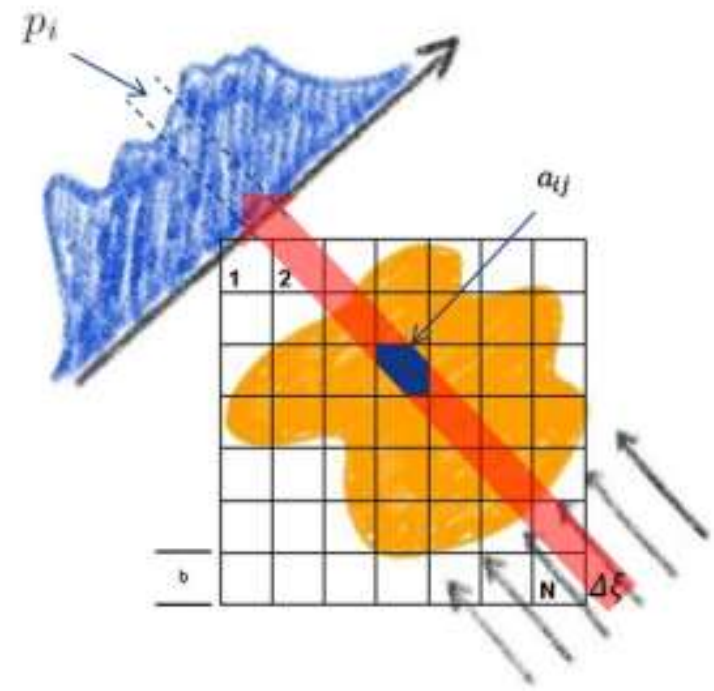

Figure (3): Demonstration of a single beam sum passing through an object [20].

The method of image reconstruction using the ART algorithm is sum up in the following steps [20]:

1. An initial guess image $\left(X^{\text {current }}\right)$ is suggested.

2. The initial guess image is corrected by employing Eq. (6) [1]:

$X^{\text {next }}$

$=X^{\text {current }}-\lambda \frac{A_{i} X^{\text {current }}-p_{i}}{\left\|A_{i}\right\|^{2}} A_{i}{ }^{T}$..

In this equation $(\lambda)$ is the relaxation parameter, which is usually chosen to be $(1),\left(p_{i}\right)$ are the measured projections, and $\left(A_{i}\right)$ is the contribution factor of the matrix along the $i$ th ray. $\left\|A_{i}\right\|^{2}=$ $\sum_{j} a_{i j}{ }^{2}$ Is the squared sum of the "contribution factors" for the $i$ th ray.

3. The previous steps are repeated starting from the second step, depending on the number of iterations.

\section{Region Growing}

Region Growing is a segmentation process that groups pixels or sub-regions in an image, into larger regions based on predefined criteria. Homogeneity of regions is the main segmentation condition in the region growing process. The criteria for homogeneity could be assigned according to; a gray level value, a pre-specified color, a distinguished shape, or any other pre-defined criteria [21].
The Region growing segmentation process could be categorized as [22]:

1. A process of merging pixels into regions.

2. A process, which splits the image pixels into regions.

3. A process which both splits-and-merges the regions in an iterative search scheme.

Region merging process recursively merges regions that are similar [23]. The merging process forms initial regions in the image, then for each region, a test is carried out to consider its adjacent regions, and see if they are similar, if so they are merged. This process is repeated until no more regions are merged [24].

Region splitting recursively divides regions that are Heterogeneous [22]. The rudimentary idea of region splitting is to break the image into a set of disjoint regions, which are coherent within themselves. This is achieved by initially taking the image as a whole to be the area of interest, deciding if all pixels contained in the region satisfy some similarity constraint, if so then the area of interest corresponds to a region in the image, if not the area of interest, is split into four equal sub-areas, and each of the sub-areas is considered as the area of interest in turn. This process continues until no further splitting occurs [25].

Splitting or merging might not produce good results when applied separately. Better results are obtained by interleaving merge and split operations [24]. Split and merge iteratively split and merge regions to form the best segmentation. This process divides an image into a set of arbitrary unconnected regions and then merges the regions in an attempt to satisfy a pre-defined constraint [23].

\subsection{Seeded Region Growing}

Seeded region growing is a semi-automatic region merging method [22]. In which the region starts with one or several seeds, each consisting of one or several pixels. The seeds are manually or automatically positioned inside the region of interest (ROI). In an intensity-based seeded region growing, the intensity of the pixels neighboring the seed is compared to the seed intensity, and if the intensity is within a specified threshold value, the neighboring pixels are added to the growing region. The growing and comparison continue with the only change that the growing region mean intensity is compared to the neighboring pixel intensity. When no pixels neighboring the growing region have an intensity within the predefined threshold value, the growth stops and the algorithm is completed [26].

There are several ways of initiating seeds. The easiest approach is to only initiate one single seed per ROI consisting of one pixel [26].

There are two parameters employed in the region growing process: the initial seed point and the target threshold ). The iterative seeded region growing procedure is as follows [27]:

1. A seed point is selected.

2. The difference between the pixel intensity value of the initial seed point and the pixel intensity values of its neighboring points are computed if the difference is smaller than the threshold that was 
initially defined, the neighboring point is classified into a cluster called .

3. The boundary of the cluster is recomputed, and those newly recomputed boundary points are set as new seed points.

4. Steps 2 and 3 are repeated, until the targeted threshold is reached.
The basic concept of the region growing image segmentation algorithm is illustrated in Fig. 4, and it shows the region growing process that begins with the seed point, and grows by adding neighbor pixels that satisfy the similarity constraint. This process is repeated until all pixels belong to some region.

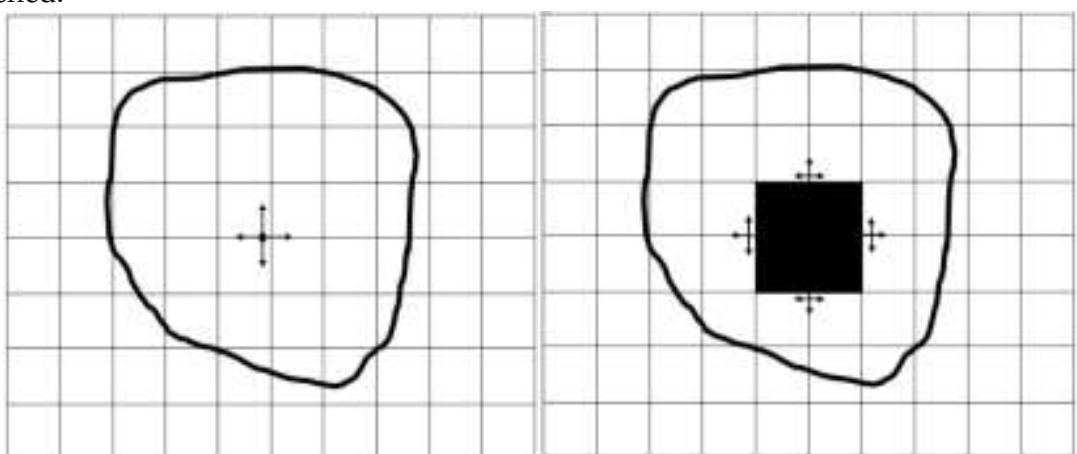

(a) (b)

Figure (4) Seeded region growing (a) First step in region growing (b) The growing process.

The segmentation process produces a segmented image and a binary image from the original input. This black and white image represents the location of the generated seeds and it is called a mask image, because it is employed as a mask on the original image during the reconstruction process, to reconstruct the desired pixels, which are selected in the seeding process. The mask binary image of three different shapes of phantoms is illustrated as shown in Fig. 5.
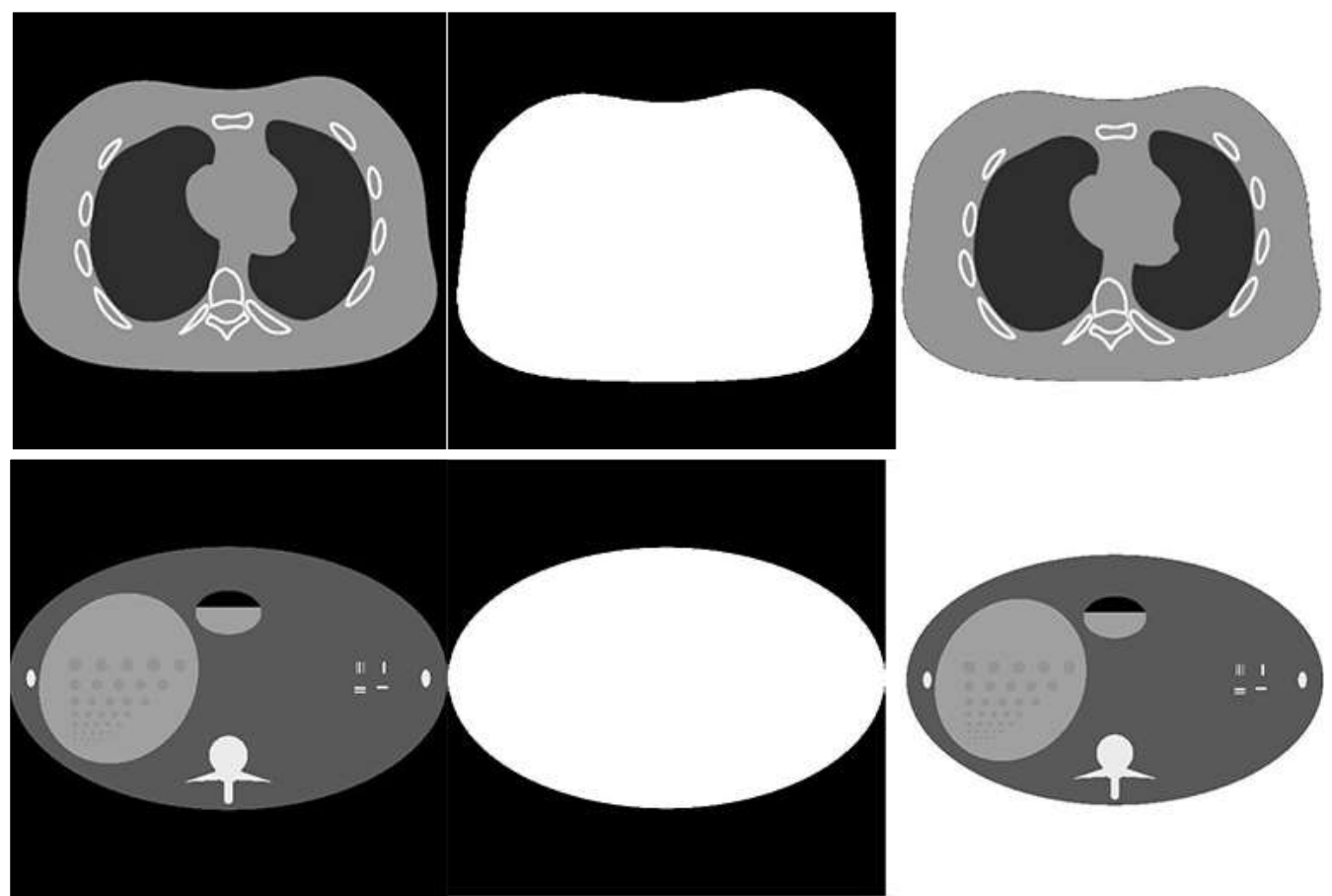

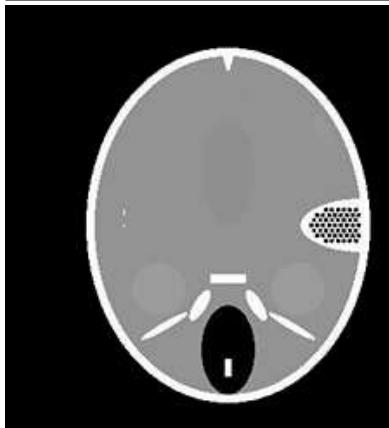

(a)

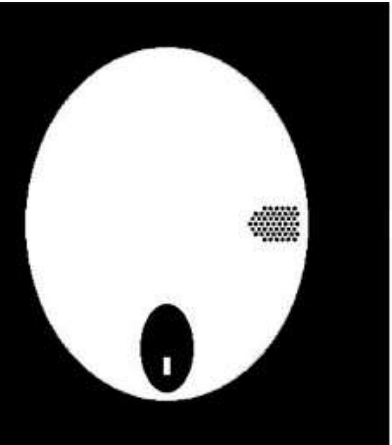

(b)

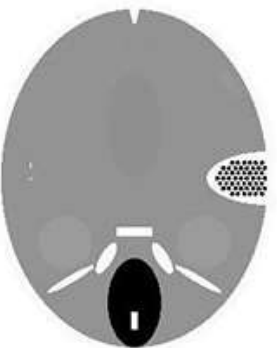

(c)

Figure (5) Phantom images (a) Original image, (b) Mask image, (c) Segmented Image. 
These figures show three different phantoms, with three different shapes that illustrate the adaptive abilities of the seeded region growing method in the segmentation process. The size of the mask and segmented image produced from the segmentation process, are the same as the original image.

\section{Computer Simulation of the Enhanced Reconstruction Algorithms}

In this paper, the reconstruction time of the iterative ART algorithm is reduced by; pre-processing the input phantom image using the seeded region growing process. Executing this process will minimize the number of pixels to be reconstructed in the iterative algorithm. The percentage of time reduction and the quality of the reconstructed image are evaluated also. This work is simulated using MATLAB. The following steps are employed to implement this enhancement:

1. A Shepp-logan head phantom is generated to be used as an input test image.

2. The seed point and the initial threshold value are chosen, to employ the seeded region-growing algorithm on the test image. This step will generate a segmented test image and a binary mask that is costumed to the segmented region. This mask will restrain the following reconstruction process, and constrict its operation, to reconstruct the desired pixels that exist where the mask equals (1), and ignore all pixels that exist where mask equals (0).

3. Projections of the segmented image are measured.

4. An initial guess image is generated, to be used for the iterative reconstruction process. The size of the generated image is the same as the input test image.

5. The relaxation parameter $(\lambda)$ is specified, and the number of iterations for the iterative reconstruction process.

6. The calculated projections for the initial guess image are stimulated.

7. Iterative reconstruction processes of the (ART) algorithm is performed. This algorithm starts by comparing the calculated and the measured projections to obtain the correction, which is then used to update the initial guess image.

8. The process of iterative reconstruction will update the image of each algorithm depending on the number of iterations specified by step (5).

9. Parameters that measure the quality of the reconstructed image are calculated as shown below:

a. Normalized mean square error (NMSE): This parameter is the summation of the squared difference values, divided by the size of the image. It is computed using Eq. (7). The normalized mean squared error is computed using Eq. (8) [28].

\section{$\mathrm{MSE}=$}

$\frac{1}{M N} \sum_{X=1}^{M} \sum_{Y=1}^{N}\left[I(X, Y)-I^{\prime}(X, Y)\right]^{2} \ldots \ldots(7)$
$\mathrm{NMSE}=\frac{\left\|\mathrm{I}(\mathrm{x}, \mathrm{y})-\mathrm{I}^{\prime}(\mathrm{x}, \mathrm{y})\right\|^{2}}{\|\mathrm{I}(\mathrm{x}, \mathrm{y})\|^{2}}$

In these equations, $\mathrm{I}(\mathrm{X}, \mathrm{Y})$ represents the image before the reconstruction, $I^{\prime}(X, Y)$ is the image after the reconstruction process, while $(\mathrm{M}, \mathrm{N})$ are the image dimensions.

b. Root mean squared error (RMSE): is a quadratic scoring rule that also measures the average magnitude of the error. It is the square root of the average of squared differences among the images before reconstruction, and after the reconstruction process as shown in Eq. (9) [29].

RMSE $=$

$\sqrt{\frac{1}{M N} \sum_{X=1}^{M} \sum_{Y=1}^{N}\left[I(X, Y)-I^{\prime}(X, Y)\right]^{2}}$

c. Peak signal to noise ratio (PSNR): signifies measuring the peak error, and it is implemented in Eq. (10)

$\operatorname{PSNR}=$

$10 \log \frac{255}{\sqrt{\left(\frac{1}{M N} \sum_{X=1}^{M} \sum_{Y=1}^{N}\left[I(X, Y)-I^{\prime}(X, Y)\right]^{2}\right)}} \ldots \ldots$.

The value of the mean square error is used in the PSNR equation. The smaller this value the less error, as noticed from the transposed relationship between these indices. Reasonably, a higher PSNR value is better since it involves a higher ratio of signal to noise. The original image is the signal, and the noise represents the error that occurs in the reconstruction [29].

d. Structural content (SC): This measure ascertains the degree of similarity between images. It is founded on minute regions in the image enclosing a significantly depleted level of physical information. The higher the existence of such regions in the images, the further the increase in the similarity, and it is computed as demonstrated in Eq. (11) [28].

$\mathrm{SC}=\frac{\sum_{X=1}^{M} \sum_{Y=1}^{N} I(X, Y)^{2}}{\sum_{X=1}^{M} \sum_{Y=1}^{N} I^{\prime}(X, Y)^{2}}$

e. Maximum difference (MD): provides the maximum of the error signal (i.e. difference between the reconstructed and original image). The higher the value of the maximum difference, the poorer the quality of the image. It is computed using Eq. (12) [29].

$\mathrm{MD}=\max \left(\left|I(X, Y)-I^{\prime}(X, Y)\right|\right) \ldots \ldots$.

f. Normalized absolute error (NAE): this measure represents a numerical difference between the primary image and the reconstructed image. A higher value of this measure shows that the image is of poor quality, it is computed using Eq. (13) [28]. 
$\mathrm{NAE}=\frac{\sum_{X=0}^{M-1} \sum_{Y=0}^{N-1}\left|I(X, Y)-I^{\prime}(X, Y)\right|}{\sum_{X=0}^{M-1} \sum_{Y=0}^{N-1}|I(X, Y)|}$

\section{Simulation Results and Discussions}

The objective of CT imaging is to reconstruct cross-sectional images of the scanned object, from the distribution of the X-ray attenuation coefficient within the object, which signifies the measured projections data.

Results of the simulated enhanced algorithm are depicted, to demonstrate the effectiveness of our approach regarding the time reduction and the improvement of the image quality indices. The prolonged reconstruction time of this algorithm is one of the major drawbacks that prohibit the application of this approach in medical image reconstruction despite its greater advantages over the analytical approach.

The results of the simulation employed the proposed Shepp-Logan head phantom, to test and carry out the simulated algorithm. This phantom is generated using a user-defined matrix; each row of the matrix indicates an ellipsoid in the image. The matrix is used to create a phantom that consists of ten columns, with each column containing a different parameter for the ellipsoids. Fig. 6 shows a $(256 \mathrm{x}$ 256) Shepp-Logan head phantom.

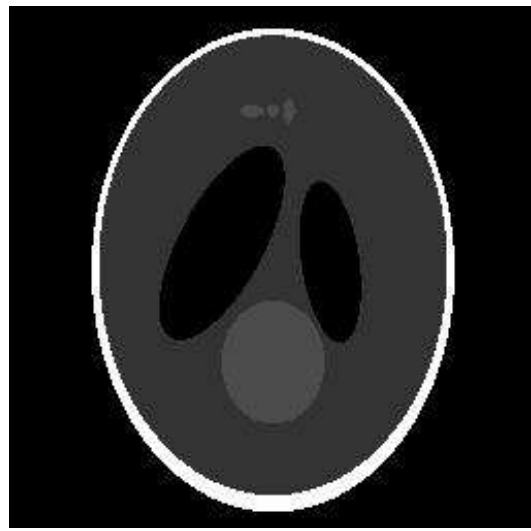

Figure (6): Shepp-logan phantom.

The computer simulation of the iterative reconstruction algorithm (ART) using a CT scanner, begins with specifying the number of pixels needed to generate the head phantom. The number of acquired projections is also specified, and it is (400) projections takin at $\left(360^{\circ}\right)$ degrees around the scanned object. Initial seeds and the initial gray level, are selected for this image to be used in the seeded region-growing algorithm. This image segmentation algorithm will eliminate all the unnecessary (background) pixels in the test image, to reduce the reconstruction time as shown in Fig. 7.

This algorithm will also generate a binary mask that is the same size as the input test image as shown in Fig. 8. This mask is used in the succeeding reconstruction steps. The use of this mask will force the reconstruction process to ignore all pixels values for pixels in the locations where the mask contains 0 's (black) and to process all pixel values in the test image, for pixels in locations where the mask contains 1's (white).

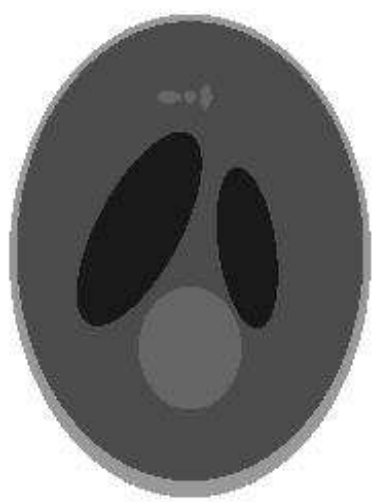

Figure (7) The segmented Shepp-Logan phantom.

The next step in the reconstruction process is to acquire the measured projections data for the test head phantom image. This step will produce (400) projections. Those projections are, obtained to simulate the motion of a CT scanner rotating $\left(360^{\circ}\right)$ degrees around the scanned object. A set of projections collected from different angles during a CT scan is called a sinogram, as shown in Fig. 9.

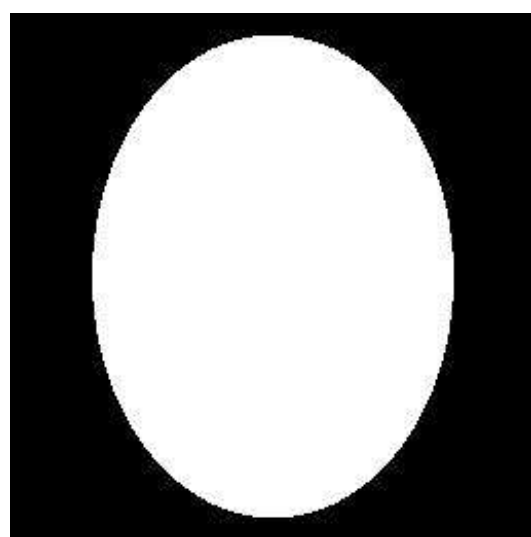

Figure (8): The binary masks of the segmented shepp-logan phantom.

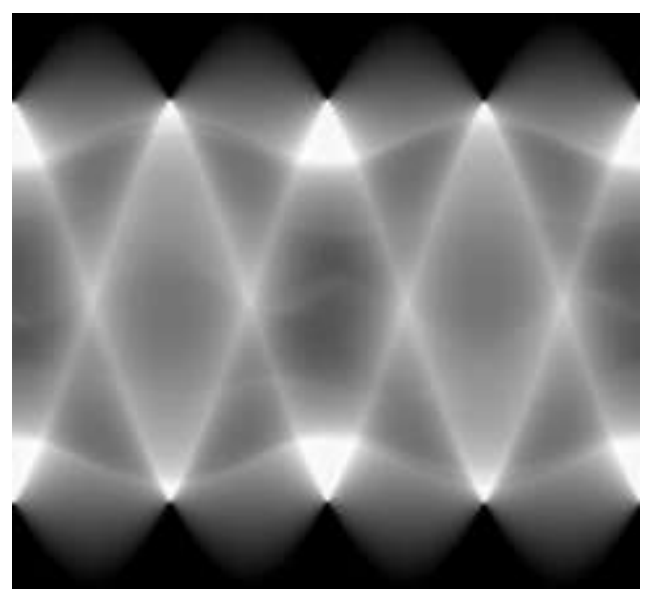

Figure (9): A sinogram of the segmented test image.

The projection generation step is accelerated due to the use of the mask that was shown in Fig. 8. This step paves the way for the subsequent reconstruction process to be performed on the wanted pixels only, thus reducing the reconstruction time. 
The following step is to generate an initial guess image, whose size is the same as the segmented test image. The relaxation parameter is chosen to be $(\lambda=1)$, and the number of iterations is (250). Increasing the number of iterations will increase the reconstruction time, but reducing it will affect the quality of the reconstructed image. Simulating the ART algorithm begins with generating a matrix of ones that is the same size as the initial guess image. This matrix is used to generate a normalization factor as indicated by Eq. (6)

The subsequent step is to start the iteration loop that begins with the first iteration and ends when the chosen number of iterations is completed. The final reconstructed image is shown in Fig. 10.

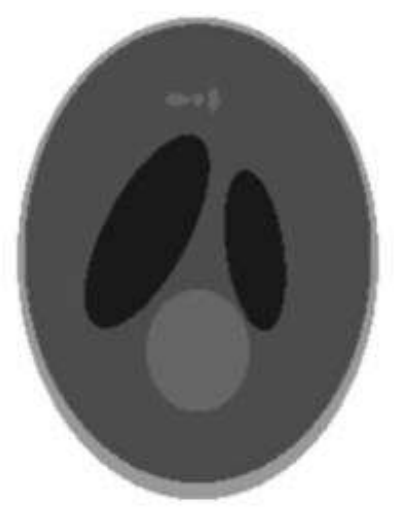

Figure (10) The reconstructed phantom image using the enhanced ART algorithm.

Different image quality indices are applied to the test images to validate the accuracy of the reconstruction process. The results are shown in Table 1.

Table (1) Image quality measurements using the enhanced ART algorithm.

\begin{tabular}{|c|c|}
\hline NMSE & $\mathbf{0 . 0 0 0 3 1 8 2 5}$ \\
\hline RMSE & 0.0178 \\
\hline PSNR & 34.9724 \\
\hline SC & 0.9988 \\
\hline MD & 0.1511 \\
\hline NAE & 0.0099 \\
\hline
\end{tabular}

Each result in Table 1 is comparing the reconstructed image with the segmented image before the reconstruction process. The reconstruction time of the enhanced ART algorithm is $(70.762696)$ seconds

Comparing these results with the results of the traditional ART algorithm. The resultant image of the original ART algorithm is shown in Fig. 11. The processing time of the original ART algorithm is (90.837304) seconds. The reconstruction time of the original algorithm far exceeds the reconstruction time of the enhanced algorithm.

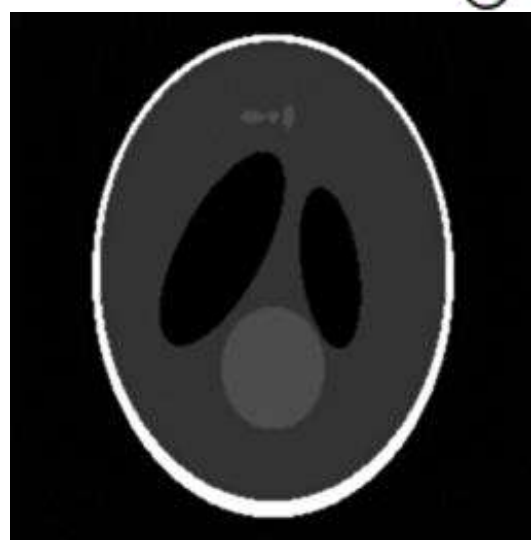

Figure (11) The reconstructed phantom image using the traditional ART algorithm.

The parameters of image quality measurements, using the traditional ART algorithm are shown in Table 2 .

Table (2) Image quality measurements using the traditional ART algorithm.

\begin{tabular}{|c|c|}
\hline NMSE & $\mathbf{0 . 0 0 0 5 3 5 4 8}$ \\
\hline RMSE & 0.0231 \\
\hline PSNR & 32.7126 \\
\hline SC & 0.9774 \\
\hline MD & 0.2197 \\
\hline NAE & 0.0544 \\
\hline
\end{tabular}

Comparing the parameters of image quality measurements between the enhanced, and the traditional unenhanced ART algorithms, which are displayed in Tables 1 and 2 . We notice that the (PSNR and SC) has increased in the enhanced ART algorithm, which is considered as a sign of good image quality. While the (NMSE, RMSE, MD, and NAE) have decreased in comparison with the traditional algorithm which indicates less error and a better reconstructed image. These results demonstrate that our approach has indeed reduced the reconstruction time as well as enhanced the quality of the image.

\section{Conclusion}

The enhancement method performed in this paper could be employed in the reconstruction of $\mathrm{CT}$ images. The proposed method is utilized to aid the reconstruction process of iterative algorithms by reducing their reconstruction time. The performance of the iterative approach was evaluated using the following image quality measures; RMSE, PSNR, MD, NAE, NMSE and SC. The main conclusions that could be drawn from the implementation of the proposed method of seeded region-based image segmentation reconstruction, when employed to the iterative ART algorithm are surmised as follows:

1. Simulating the proposed enhancement method on the iterative ART algorithm reduced the reconstruction time by $(24.8448 \%)$.

2. The PSNR and SC of the reconstructed image have improved as shown by Tables 1 and 2 when 
utilizing the proposed enhancement method to the ART algorithm. The other image quality indices (RMSE, NMSE, MD, and NAE) decreased, which indicates a better-reconstructed image with fewer errors.

This enhancement approach could be further supported by testing it on a different iterative reconstruction algorithm. Our method could also be tested on the three-dimensional cone-beam CT image reconstruction. The quest for faster reconstruction of iterative $\mathrm{CT}$ images could be further improved by suggesting alternative approaches, or by employing an analytical and iterative reconstruction process, which is known as a hybrid algorithm to further reduce the reconstruction time.

\section{References}

[1] G. Zeng, "Medical Image Reconstruction". Berlin, Heidelberg: Springer, 2010.

[2] D. Brenner and E. Hall, "Risks from CT Scans: Now We Have Data, What Next?", Radiology, vol. 265, no. 2, pp. 330-331, 2012. Available: 10.1148/radiol.12121248.

[3] J. Hsieh, B. Nett, Z. Yu, K. Sauer, J. Thibault and C. Bouman, "Recent Advances in CT Image Reconstruction", Current Radiology Reports, vol. 1, no. 1, pp. 39-51, 2013. Available: 10.1007/s40134-012-0003-7.

[4] M. Beister, D. Kolditz and W. Kalender, "Iterative reconstruction methods in X-ray CT", Physica Medica, vol. 28, no. 2, pp. 94-108, 2012. Available: 10.1016/j.ejmp.2012.01.003.

[5] M. Willemink and P. Noël, "The evolution of image reconstruction for CT from filtered back projection to artificial intelligence", European Radiology, vol. 29, no. 5, pp. 2185-2195, 2018. Available: 10.1007/s00330-018-5810-7.

[6] S. Ha and K. Mueller, "Metal Artifact Reduction in X-ray CT via Ray Profile Correction", Applied Sciences, vol. 10, no. 1, p. 66, 2019. Available: 10.3390/app10010066.

[7] T. Van Hemelryck, S. Wuyts, M. Goossens, J. Batenburg Kees and J. Sijbers, "ITERATIVE RECONSTRUCTIN ALGORITHMS The implementation of iterative reconstruction algorithms in MATLAB", 2007.

[8] Y. Chen, Z. Wang, W. Zhao and X. Yang, "Liver Segmentation from CT Images Based on Region Growing Method", 2009 3rd International Conference on Bioinformatics and Biomedical Engineering, pp. 1 - 4, 2009. Available: 10.1109/icbbe.2009.5163018.

[9] M. A.Brooks, "A Survey of Algebraic Algorithms in Computerized Tomography", Undergraduate, University of Ontario Institute of Technology, 2010.

[10] N. Mesanovic, M. Grgic, H. Huseinagic, M. Males, E. Skejic and M. Smajlovic, "Automatic CT image segmentation of the lungs with region growing algorithm", 18th International Conference on Systems, Signals and Image Processing-IWSSIP, pp. 395-400, 2019.
[11] K. Shilpa and K. R, "Image Segmentation and Region Growing Algorithm", Int. J. Comput. Technol, vol. 2, 2012.

[12] C. Panchasara and A. Joglekar, "Application of Image Segmentation Techniques on Medical Reports", International Journal of Computer Science and Information Technologies, vol. 6, no. 3, pp. 2931-2933, 2015.

[13] N. Hussein Fallooh, "Evaluation and Simulation of the Image Reconstruction Algorithms in ConeBeam Computed Tomography”, Undergraduate, Al-Nahrain University, 2015.

[14] M. Aurumskjöld, "Optimisation of image quality and radiation dose in computed tomography using iterative image reconstruction", MSc, Lund University, 2017.

[15] B. Ait Skourt, A. El Hassani and A. Majda, "Lung CT Image Segmentation Using Deep Neural Networks", Procedia Computer Science, vol. 127, pp. 109-113, 2018. Available: 10.1016/j.procs.2018.01.104.

[16] H. Kawashima, K. Ichikawa, K. Matsubara, H. Nagata, T. Takata and S. Kobayashi, "Quality evaluation of image-based iterative reconstruction for CT: Comparison with hybrid iterative reconstruction", Journal of Applied Clinical Medical Physics, 2019. Available: 10.1002/acm2.12597.

[17] Mueller, Klaus \& Yagel, Roni \& J. Wheller, John, "Fast and accurate projection algorithm for 3D cone-beam reconstruction with the Algebraic Reconstruction Technique (ART)", Proceedings of SPIE - The International Society for Optical Engineering, 1998. Avaliable: 10.1117/12.317078.

[18] A. Kak and M. Slaney, Principles of computerized tomographic imaging. Philadelphia (Pa.): Society for Industrial and Applied Mathematics, 2001.

[19] L. Geyer et al., "State of the Art: Iterative CT Reconstruction Techniques", Radiology, vol. 276, no. 2, pp. 339-357, 2015. Available: 10.1148/radiol.2015132766.

[20] T. Buzug, "Introduction to Computed Tomography”. Dordrecht: Springer, 2008.

[21] M. T. Wanjari, K. D. Kalaskar and D. P. Dhore, "Document Image Segmentation using Region Based Methods", International Journal of Computing Science and Information Technology, vol. 3, no. 3, pp. 01-08, 2015.

[22] S. Uchida, "Image processing and recognition for biological images", Development, Growth \& Differentiation, vol. 55, no. 4, pp. 523-549, 2013. Available: 10.1111/dgd.12054.

[23] C. A. Bouman, "EE637: Digital Image Processing I", Engineering.purdue.edu, 2019. [Online]. Available: https://engineering.purdue.edu/ bouman/ece63 7/notes/pdf/. [Accessed: 16- Dec- 2019].

[24] C. Steger, M. Ulrich and C. Wiedemann, Machine vision algorithms and applications, 2nd ed. WileyVCH, 2017.

[25] D. Marshall, "Region Splitting", Users.cs.cf.ac.uk, 2019. [Online]. Available: 
https://users.cs.cf.ac.uk/Dave.Marshall/Vision_1 ecture/node34.html. [Accessed: 16- Dec- 2019].

[26] S. Zhou, D. Rueckert and G. Fichtinger, Handbook of Medical Image Computing and Computer Assisted Intervention, 1st ed. 2019.

[27] C. Huang, L. Luo, P. Lee, J. Lai, W. Wang and S. Lin, "Efficient Segmentation Algorithm for 3D Bone Models Construction on Medical Images", Journal of Medical and Biological Engineering, vol. 31, 2011. Available: 0.5405/jmbe. 734 .

[28] M. Brambilla, B. Cannillo, M. Dominietto, L. Leva, C. Secco and E. Inglese, "Characterization of ordered-subsets expectation maximization with $3 \mathrm{~d}$ post-reconstruction gauss filtering and comparison with filtered backprojection in $99 \mathrm{~m}$ Tc SPECT", Annals of Nuclear Medicine, vol. 19, no. 2, pp. 75-82, 2005. Available: $10.1007 /$ bf03027384.
[29] W. Wang and Y. Lu, "Analysis of the Mean Absolute Error (MAE) and the Root Mean Square Error (RMSE) in Assessing Rounding Model", IOP Conference Series: Materials Science and Engineering, vol. 324, p. 012049, 2018. Available: 10.1088/1757-899x/324/1/012049.

[30] N. H. Fallooh Al-anbari and M. H. Ali AlHayani, "Design and Construction ThreeDimensional Head Phantom Test Image for the Algorithms of 3D Image Reconstruction", Journal of Emerging Trends in Computing and Information Sciences, vol. 6, no. 2, 2015.

[31] N. H. Fallooh Al-anbari and M. H. Ali AlHayani, "Evaluation Performance of Iterative Algorithms for 3D Image Reconstruction in Cone Beam Geometry", Al-Nahrain jounal of engineering sciences, vol. 20, no. 1, pp. 149-157, 2017. 